\title{
Structure of the C-terminal domain of transcription factor IIB from Trypanosoma brucei
}

\author{
B. Syed Ibrahima,1, Nalini Kannegantia, Gabrielle E. Rieckhofb,2, Anish Dasc, Douglas V. Laurentsd, Jennifer B. Palenchare, \\ Vivian Bellofatto', and David A. Wah",3 \\ aPublic Health Research Institute and Department of Biochemistry and Molecular Biology and 'Department of Microbiology and Molecular Genetics, New \\ Jersey Medical School, University of Medicine \& Dentistry of New Jersey, 225 Warren Street, Newark, NJ 07103; 'bThe Rockefeller University, 1230 York \\ Avenue, New York, NY 10021; dInstituto de Química Física "Rocasolano," Consejo Superior de Investigaciones Científicas, Serrano 119,28006 Madrid, Spain; \\ and eDepartment of Chemistry, 214D Mendel Science Center, Villanova University, Villanova, PA 19085
}

Edited by Robert T. Sauer, Massachusetts Institute of Technology, Cambridge, MA, and approved June 18, 2009 (received for review May 14, 2009)

In trypanosomes, the production of mRNA relies on the synthesis of the spliced leader (SL) RNA. Expression of the SL RNA is initiated at the only known RNA polymerase II promoter in these parasites. In the pathogenic trypanosome, Trypanosoma brucei, transcription factor IIB (tTFIIB) is essential for SL RNA gene transcription and cell viability, but has a highly divergent primary sequence in comparison to TFIIB in well-studied eukaryotes. Here we describe the $\mathbf{2 . 3}$ Å resolution structure of the C-terminal domain of tTFIIB (tTFIIBC). The TTFIIB $_{C}$ structure consists of 2 closely packed helical modules followed by a C-terminal extension of 32 aa. Using the structure as a guide, alanine substitutions of basic residues in regions analogous to functionally important regions of the well-studied eukaryotic TFIIB support conservation of a general mechanism of TFIIB function in eukaryotes. Strikingly, TTFIIB $_{C}$ contains additional loops and helices, and, in contrast to the highly basic DNA binding surface of human TFIIB, contains a neutral surface in the corresponding region. These attributes probably mediate trypanosomespecific interactions and have implications for the apparent bidirectional transcription by RNA polymerase II in protein-encoding gene expression in these organisms.

eukaryotic transcription | parasite | RNA polymerase II | trypanosome

T rypanosomes are flagellated protozoa, belonging to the order Kinetoplastida, that are exclusively parasitic (1). These organisms reside in insect vectors that transmit the parasites to mammals, birds, fish, and plants. Trypanosoma brucei and Trypanosoma cruzi are of particular medical concern because they cause debilitating and fatal diseases in millions of people annually in tropical regions of the world (2). Trypanosomes diverged from other eukaryotes early in evolution, and thus many biological processes that are well understood in metazoans are highly distinct in these parasites $(3,4)$. For example, little is known about how trypanosome RNA polymerase II (tRNAP-II) transcription is initiated. It is known that tRNAP-II transcribes most protein-encoding genes, which are arranged in tandem arrays, into polycistronic mRNAs (5). Thus far, the only tRNAP-II promoter that has been identified is the spliced leader (SL) RNA gene promoter (6). The SL RNA gene codes for the $\mathrm{SL}$, which is capped and added in a trans-splicing reaction to the $5^{\prime}$ end of each ORF contained in a polycistronic mRNA. The addition of an SL to every mRNA is a universal requirement in trypanosomes; therefore, understanding transcription initiation at the SL RNA gene promoter is a crucial step toward understanding the control of tRNAP-II transcription in these parasites $(4,7)$.

To date, components of the SL RNA transcriptional machinery have been studied by using genomics or biochemistry to identify candidate proteins followed by a combination of phenotypic analysis of protein function using either RNAi-silencing of endogenous protein in parasites or depletion/add back studies of in vitro transcription systems. These methods have identified several players in tRNAP-II-dependent SL RNA transcription, including trypanosome TATA-box binding protein (tTBP), transcription factors IIB, -IIA, -IIH (tTFIIB, tTFIIA, tTFIIH), and tSNAPc (8-17). However, each trypanosome factor is only distantly related to its metazoan homolog and contains regions or subunits unique to trypanosomes. Indeed, a recent study of tTFIIH using single particle electron microscopy suggests that tTFIIH has a similar structure to human TFIIH but contains trypanosome-specific subunits (18). Thus, the mechanism of transcription initiation in trypanosomes cannot be deduced from metazoan transcription systems. Our work presented here is the primary venture to understand the mechanics of the SL RNA transcriptional machinery using high-resolution structural analysis.

Trypanosome TFIIB is essential for SL RNA transcription in T. brucei $(13,14)$. tTFIIB associates with tTBP, tRNAP-II, and an SL RNA gene promoter fragment in nuclear extracts. In yeast and mammals, TFIIB binds specifically to TBP and DNA and recruits RNAP-II into a minimal preinitiation complex (reviewed in ref. 19). TFIIB consists of $\mathrm{N}$ - and C-terminal domains $(20,21)$. NMR analysis of the N-terminal domain from Pyrococcus furiosus TFIIB reveals a $\mathrm{Zn}$ ribbon motif and an extended B finger loop $(22,23)$. In the cocrystal structure of Saccharomyces cerevisiae TFIIB and RNAP-II, the TFIIB N-terminal domain binds RNAP-II and uses the B finger loop to finely position RNAP-II at the transcription start site (24). NMR analysis of the human TFIIB C-terminal domain reveals 2 helical repeats (25). In the human and Pyrococcus woesei TFIIB/TBP/ DNA ternary complexes, the TFIIB C-terminal domain recognizes TBP and the promoter (26-29). The C-terminal domain of the trypanosome TFIIB (tTFIIB ${ }_{C}$ ) contains 1 loosely conserved repeat module followed by a trypanosome-specific region (13, 14). Here, we report the $2.3 \AA$ resolution structure of tTFIIB $_{C}$. The TFIIB $_{C}$ structure reveals 2 , closely packed helical modules followed by a C-terminal extension of 32 aa. The trypanosomespecific region comprises the second helical module and the $\mathrm{C}$-terminal extension. The overall $\mathrm{tTFIIB}_{\mathrm{C}}$ structure is similar to other $\mathrm{TFIIB}_{\mathrm{C}}$ structures (25-29), but contains additional loops and helices. In contrast to the highly basic DNA binding surface of human TFIIB, the structure reveals a neutral surface in the

Author contributions: J.B.P., V.B., and D.A.W. designed research; B.S.I., N.K., G.E.R., and D.A.W. performed research; B.S.I., N.K., A.D., and J.B.P. contributed new reagents/analytic tools; B.S.I., D.V.L., V.B., and D.A.W. analyzed data; and G.E.R., D.V.L., V.B., and D.A.W. wrote the paper.

The authors declare no conflict of interest.

This article is a PNAS Direct Submission.

Data deposition: The atomic coordinates and structure factors have been deposited in the Protein Data Bank, www.pdb.org (PDB ID code 3H4C).

1Present address: Biology Department, Brookhaven National Laboratory, Upton, NY 11973.

2Present address: New York Academy of Sciences, 7 World Trade Center, New York, NY 10007

${ }^{3}$ To whom correspondence should be addressed. E-mail: wahda@umdnj.edu.

This article contains supporting information online at www.pnas.org/cgi/content/full/ 0904309106/DCSupplemental. 


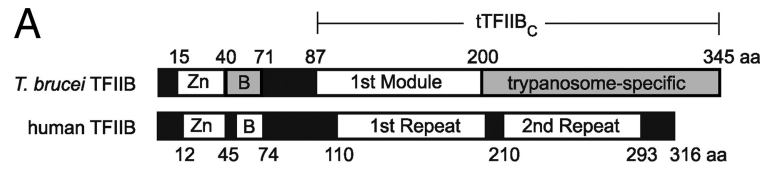

B $\quad$ tTFIIB $_{C}$
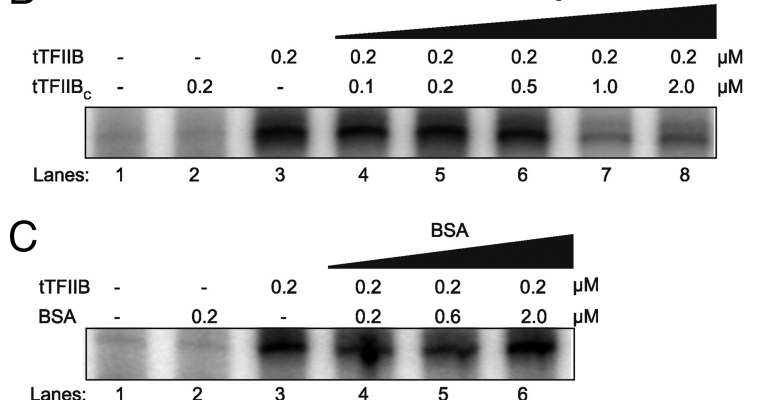

Fig. 1. Primary structure and function of tTFIIB and tTFIIB $B_{C}(A)$ Schematic of T. bruce i and human TFIIB amino acid sequence alignment. The $\mathrm{Zn}$ ribbon and the first module/first repeat are well conserved between trypanosomes and humans. The $B$ finger and the trypanosome-specific/second repeat are not well conserved (gray background). TTFIIB $_{C}$ is 259 residues long, comprising the first module and the trypanosome-specific region (bracketed). (B) Activity of tTFIIB and TTFIIB $_{C}$ in in vitro SL RNA transcription assays. Transcription activity in tTFIIB-depleted extracts (lane 1) could not be restored by $0.2 \mu \mathrm{M}$ tTFIIB $_{C}$ (lane 2), whereas tTFIIB restored maximally at that concentration (lane 3). tTFIIB $_{C}$ maximally inhibited restoration by tTFIIB when added in 5-fold molar excess (lanes 4-8). (C) In control assays, BSA did not restore transcription (lane 2) and did not inhibit restoration by tTFIIB (lanes $4-6$ ).

corresponding region. These attributes are probably functionally important, mediating trypanosome-specific interactions in a preinitiation complex.

\section{Results}

tTFIIB and tTFIIB Are Stably Folded Monomers. We performed limited proteolysis on recombinant tTFIIB to identify a stable fragment amenable to crystallization, because the full-length protein was refractory to crystallization. The human TFIIB C-terminal domain forms a protease-resistant core $(20,21)$. In contrast, tTFIIB was highly protease sensitive and did not yield any stable fragments ( Fig. S1). Therefore, we defined the tTFIIB C-terminal domain $\left(\right.$ tTFIIB $\left._{C}\right)$ by comparing the amino acid sequence of tTFIIB with those of crystal structures of human and archaeal TFIIB C-terminal domains as shown schematically (Fig. $1 A$ ). tTFIIB $_{\mathrm{C}}$, which comprises the first module and the trypanosome-specific region (residues 87-345), was expressed and purified.

Because the limited proteolysis results suggested that tTFIIB and tTFIIB $_{\mathrm{C}}$ might not be stably folded, we characterized both proteins biophysically. In gel filtration experiments, each protein eluted as a single peak at a position between its calculated dimer and monomer mass (Fig. S2A). This is consistent with the extended monomer conformation of TFIIB and TFIIB $_{C}$ from other organisms (23-25). We found that the fluorescence spectrum of native tTFIIB was blue-shifted when compared to the spectrum under denaturing conditions, suggesting hydrophobic burial of Trp-260 in the native protein. Because Trp-260 is the only tryptophan in tTFIIB and resides in the C-terminal domain, unfolding of both tTFIIB and TFFIB $_{\mathrm{C}}$ could be monitored by fluorescence. Denaturant-induced unfolding of tTFIIB and tTFIIB $_{C}$ occurred in a cooperative and reversible fashion with a half-maximal $\mathrm{C}_{\mathrm{m}}$ of unfolding between 2 and $3 \mathrm{M}$ guanidinium chloride (Fig. S2B). tTFIIB $_{C}$ appears to be slightly more stable than full-length tTFIIB $\left(\mathrm{C}_{\mathrm{m}}=2.9 \mathrm{M}\right.$ for tTFIIB $_{\mathrm{C}}$ versus $2.7 \mathrm{M}$ for tTFIIB). Taken together, these results indicate that fulllength tTFIIB and TFFIIB $_{C}$ are stably folded monomers.
Activity of Purified tTFIIB and tTFIIB $_{\mathbf{c}}$ by in Vitro Transcription. We showed previously that TTFIIB expressed and purified from Escherichia coli restores transcription in vitro when added to tTFIIB-immunodepleted nuclear extracts of T. brucei (14). Using this system, tTFIIB restored transcription maximally at $0.2 \mu \mathrm{M}$ (Fig. $1 B$, lane 3, and Fig. S3). By contrast, TTFIIB $_{\mathrm{C}}$ was unable to restore transcription (Fig. $1 B$, lane 2). Notably, tTFIIB $_{C}$ had a trans-dominant negative effect on transcription. TTFIIB $_{C}$ inhibited transcription in the presence of full-length tTFIIB when tTFIIB $_{C}$ was added in 5-fold molar excess (Fig. $1 B$, lane 7). This effect is specific, as transcription by tTFIIB was unaffected by molar excess of BSA (Fig. 1C). We hypothesize that TFFIIB $_{C}$ is able to bind to proteins and/or DNA in the preinitiation complex but requires the $\mathrm{N}$-terminal domain to stimulate transcription, consistent with previous studies of human $\mathrm{TFIIB}_{\mathrm{C}}(21)$.

Structure of tTFIIB $\mathrm{B}_{\mathrm{C}}$ and Comparison to Human tTFIIB $_{\mathrm{c}}$. We crystallized and solved the structure of $\mathrm{tTFIIB}_{\mathrm{C}}$ at $2.3 \AA$ resolution $\left(\mathrm{R}_{\mathrm{work}}=\right.$ $20.3 \% ; \mathrm{R}_{\text {free }}=24.9 \%$ ) (Table S1 and Table S2). The tTFIIB $_{\mathrm{C}}$ structure reveals 2 , closely packed helical modules followed by a C-terminal extension of 32 aa (Fig. 2A). The trypanosome-specific region comprises the second helical module and the C-terminal extension. The first helical module contains 6 helices $(\mathrm{H} 1-\mathrm{H} 6)$, while the second module has 8 helices $\left(\mathrm{H} 1^{\prime}-\mathrm{H} 6^{\prime}\right.$ and $23_{10}$ helices, $\mathrm{H} 2^{\prime} \mathrm{A}$ and $\mathrm{H}^{\prime} \mathrm{A}$ ). Both helical modules contain the canonical 5-helix cyclin fold characteristic of TFIIB proteins (helices H1-H5 in the first module and $\mathrm{H} 1^{\prime}-\mathrm{H} 5^{\prime}$ in the second module). The cyclin fold in each module aligns well to the cyclin folds of human TFIIB $_{C}$ (rmsd $=1.8 \AA$ on 90 of 96 matched $\mathrm{C}_{\alpha}$ atoms for the first module; rmsd $=2.3 \AA$ on 86 of 100 matched $C_{\alpha}$ atoms for the second module). The cyclin fold of the second module has a slightly greater deviation from the human cyclin folds owing to divergent regions discussed below. The 32-residue C-terminal extension of tTFIIB $_{C}$ (residues 314-345) is not visible in the crystal, suggesting that it is unstructured. The prevalence of Pro, Glu, Ser, and Lys residues in the C-terminal extension of the $\operatorname{tTFIIB}_{\mathrm{C}}$ is consistent with this conclusion (30).

The overall tTFIIB $_{C}$ structure is similar to other TFIIB $_{C}$ structures (25-29); however, the additional loops and helices between the modules and within the second module of tTFIIB $_{C}$ explain why primary sequence analysis did not reveal the second cyclin fold $(13,14)$ (Fig. $2 A$ and $B$ ). For instance, the first module has an additional helix $\mathrm{H} 6$ that links the 2 modules together by connecting $\mathrm{H} 5$ and $\mathrm{H} 1{ }^{\prime}$. Helix $\mathrm{H} 6$ essentially forms a bent, 24-residue helix with $\mathrm{H} 1^{\prime}$ of the second module (Fig. $2 \mathrm{~A}$, blue arrow). In human TFIIB $_{\mathrm{C}}$, the helical repeats are connected by a short, random-coil segment that interacts with TBP (26) (Fig. $2 B$, blue arrow). In tTFIIB $_{C}$, the presence of a helix at this position instead of a random coil creates a larger surface that would allow for additional contacts with tTBP.

The second module in TFIIB $_{C}$ contains 2 significant insertions within its cyclin fold that are not observed in other TFIIB structures. The first insertion is the helix $\mathrm{H} 2^{\prime} \mathrm{A}$, which is a short $3_{10}$ helix inserted into the loop connecting helices $\mathrm{H} 2^{\prime}$ and $\mathrm{H}^{\prime}$. The second insertion is a much longer segment within the loop between helices $\mathrm{H}^{\prime}$ ' and $\mathrm{H}^{\prime}$ '. This insertion consists of another $3_{10}$ helix, H3' A, that is followed by a 13-residue linker (Fig. $2 \mathrm{~A}$, black arrow). In the $\mathrm{tTFIIB}_{\mathrm{C}}$ structure, $\mathrm{H}^{\prime} \mathrm{A}$ veers $\approx 90^{\circ}$ from H3' and away from H4'. The linker (residues 162-174), which connects $\mathrm{H}^{\prime}{ }^{\prime} \mathrm{A}$ to $\mathrm{H}^{\prime}{ }^{\prime}$, is not visible in the electron density, suggesting that it is unstructured. T. cruzi TFIIB $_{\mathrm{C}}$ also contains a linker in this region of different length but with similar amino acid composition to $T$. brucei TFIIB (Fig. $2 C$, black arrow). There appears to be no precedent for a long unstructured segment between helices $\mathrm{H}^{\prime}$ ' and $\mathrm{H}^{\prime}{ }^{\prime}$ in either TFIIB or cyclin structures in the Protein Data Bank, as assessed by a search using the DALI structural alignment server (31). In these well-studied proteins, the region connecting $\mathrm{H}^{\prime}{ }^{\prime}$ and $\mathrm{H}^{\prime}$ ' is typically a 

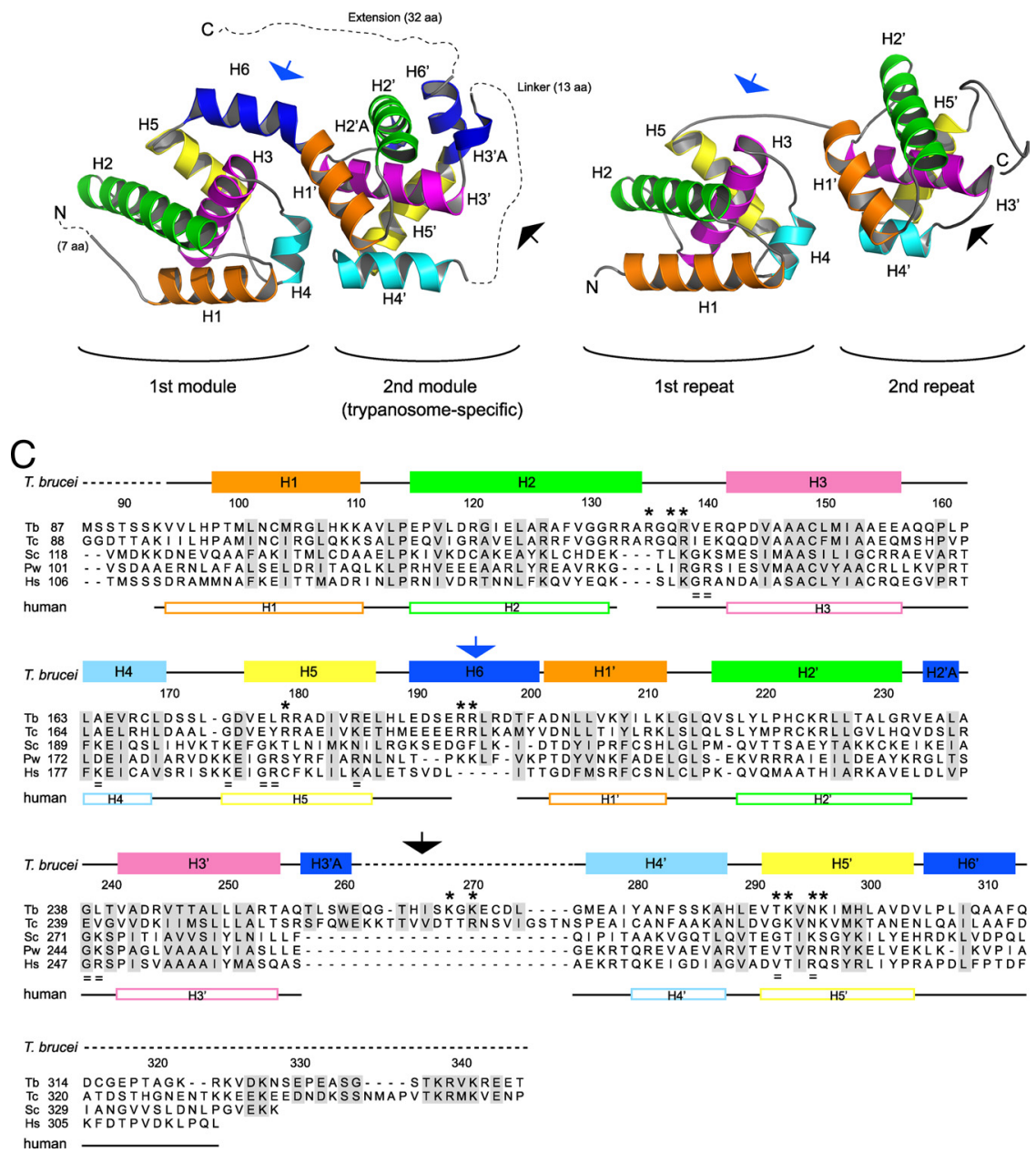

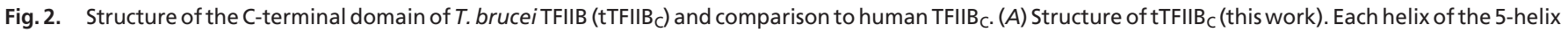

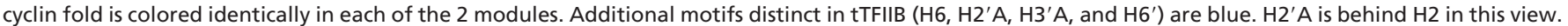
Dashed lines denote amino acids not visible in the crystal, and the number of residues is in parentheses. Arrows denote regions for comparison between tTFIIB $_{C}$ and human TFIIB ${ }_{C}$ in panel $B$ and their position in the sequence in panel $C$ as discussed in the text: Helix $\mathrm{H} 6$ (blue arrow) and the linker between $\mathrm{H}^{\prime}{ }^{\prime} \mathrm{A}$ and $\mathrm{H}^{\prime}{ }^{\prime}$ (black arrow). (B) Structure of human TFIIBC (PDB ID 1c9b) (29). (C) The amino acid sequence of tTFIIB ${ }_{C}$ (Tb; accession no. EAN76636) is aligned with that of $T$. cruzi (Tc; XP 806216), S. cerevisiae (Sc; P29055), and the sequences of known TFIIB $c$ structures from P. woesei (Pw; 1d3u), and Homo sapiens (Hs; 1c9b). Sequence gaps are denoted by dashes in panel C. TFFIIB $_{C}$ amino acid numbers and secondary structure elements are indicated above the alignment, and human TFIIB $\mathrm{B}_{\mathrm{C}}$ secondary structure elements are indicated below the alignment. Helices are depicted as boxes, intervening segments as lines, gaps in the structural alignment are blank, and residues not visible in the electron density are denoted by dashes. Amino acids that were changed to alanine in tTFIIB variants are indicated by asterisks (see Figs. 3 and 4). Structurally equivalent residues in human TFIIB that affected function when mutated are denoted by equal signs (19). Residues Asp-229 to Thr-237 are omitted from the S. cerevisiae sequence for clarity.

structured loop of 5-8 residues (Fig. $2 B$, black arrow). Unstructured protein segments often contain short recognition motifs that mediate protein-protein or protein-nucleic acid interactions (32). We predict that the TTFIIB $_{C}$ unstructured linker is functionally important, mediating trypanosome-specific interactions in the preinitiation complex.

The overall conformation of TTFIIB $_{C}$ is closer to human TFIIB $_{C}$ bound in the TFIIB $/$ TBP/DNA complex rather than to the unbound protein $(25,29)$. This allows us to predict how tTFIIB $_{C}$ might interact with DNA and tTBP. Strikingly, the electrostatic potential of the putative DNA binding surface of tTFIIB $_{\mathrm{C}}$ is markedly different from the DNA binding surface of human TFIIB $_{\mathrm{C}}$ (Fig. $3 A$ ). In the human TFIIB $_{\mathrm{C}} / \mathrm{TBP} / \mathrm{DNA}$ complex, TFIIB ${ }_{C}$ contacts DNA through a large positive surface (Fig. $3 B$ ). This surface stabilizes the TBP-induced deformation of the DNA by interacting both upstream and downstream of the TATA box. In the TFIIB ${ }_{\mathrm{C}} / \mathrm{TBP} / \mathrm{DNA}$ complex, the DNA tracks along the large positive surface of TFIIB $_{\mathrm{C}}$ (Fig. $3 B$ ). In stark contrast, the analogous surface of TFIIB $_{C}$ is relatively neutral and contains only 2 small patches of positive charge (Fig. $3 A$, Left). Thus the trypanosome TFIIB $_{\mathrm{C}} / \mathrm{TBP} / \mathrm{DNA}$ complex probably has a different conformation than the canonical ternary complex.

tTFIIB $_{C}$ likely interacts with DNA through the 2 small positive patches found on the electrostatic surface of the structure (Fig. $3 A$, Left). The first patch corresponds to the loop between helices $\mathrm{H} 2$ and $\mathrm{H} 3$ (Fig. 3C, Left). In human TFIIB $_{\mathrm{C}}$, the loop between helices $\mathrm{H} 2$ and $\mathrm{H} 3$, known as the recognition loop, binds to the DNA downstream of the TATA box at the downstream TFIIB recognition element $\left(\mathrm{BRE}^{\mathrm{D}}\right)$ (Fig. 3 A-C, Right) $(26,29)$. tTFIIB $_{\mathrm{C}}$ contains a similar loop that is preceded by an additional helical turn at the C-terminal end of H2 (Fig. 3C, Left, green arrow). This helical turn extends out from the first module into solvent, placing basic residues in the loop, namely Arg-135 and Arg-138, in position to interact 
A

T. brucei TFIIB $\quad$ human TFIIB $_{\mathrm{C}}$
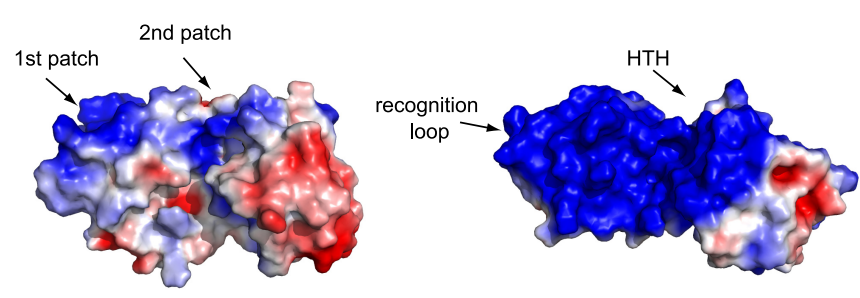

B
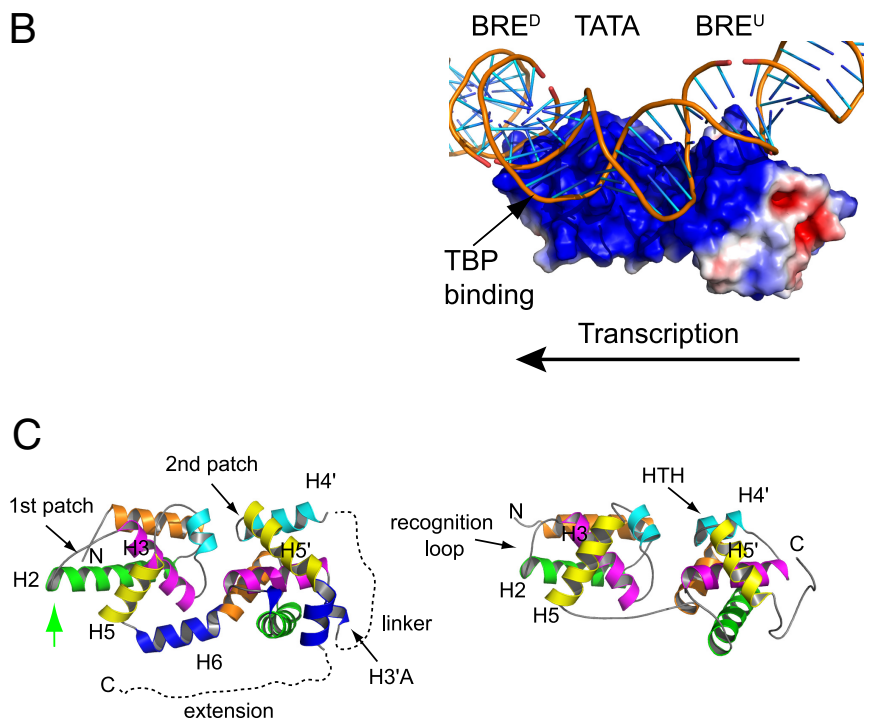

Fig. 3. Putative DNA binding surface and electrostatic potential surface properties of $\mathrm{TTFIIB}_{C}$ compared to human TFIIB $\mathrm{B}_{C}$. $(A)$ The putative DNA binding face of $\mathrm{TTFIIB}_{C}$ (Left) reveals a more neutral surface than the highly basic DNA binding surface of human $\operatorname{TTFIIB}_{C}(R i g h t)$. Areas colored in blue, white, and red denote positive, neutral, and negative potential contoured at $+3,0,-3 \mathrm{kT} / \mathrm{e}$, respectively. Black arrows in panels $A$ and $C$ indicate the basic patches in $\operatorname{TTFIIB}_{C}$ and the corresponding regions (recognition loop and HTH motif) in human TFIIB $_{C}$. (B) In the human TFIIB $C$ TRBP/DNA complex, TFIIB ${ }_{C}$ contacts the DNA at $B R E^{D}$ and $B^{\prime} E^{U}$ through its basic surface (29). TBP, which binds the minor groove, is omitted for clarity. (C) Ribbon diagram of putative DNA binding surface. The green arrow indicates the additional turn on $\mathrm{H} 2$ unique to the trypanosome protein. View in this figure is rotated $180^{\circ}$ around the horizontal axis relative to Fig. 2 .

with the DNA. Thus, tTFIIB $_{C}$ may use the extra helical turn of $\mathrm{H} 2$ as well as its loop for DNA binding.

The second positive patch in $\mathrm{TTFIIB}_{\mathrm{C}}$ corresponds to $\mathrm{H}^{\prime}{ }^{\prime}$ and $\mathrm{H}^{\prime}$ (Fig. $3 A$ and $C$, Left). In human $\mathrm{TFIIB}_{\mathrm{C}}, \mathrm{H}^{\prime}$ and $\mathrm{H}^{\prime}$ ' form a helix-turn-helix $(\mathrm{HTH})$ motif that binds the DNA upstream of the TATA box at the upstream TFIIB recognition element (BRE ${ }^{\mathrm{U}}$ ) (Fig. $3 B$ and $C$, Right) $(29,33)$. In the HTH motif, helix $\mathrm{H} 4$ ' uses a conserved glutamine to stabilize a conserved arginine in $\mathrm{H}^{\prime}$ ' that contacts DNA (29). Interestingly, tTFIIB $_{\mathrm{C}}$ lacks these conserved residues. Moreover, $\mathrm{H}^{\prime}$ in $\mathrm{tTFIIB}_{\mathrm{C}}$ is 1 turn longer than the first helix of the HTH motif of human TFIIB $_{\mathrm{C}}$. This would not allow $\mathrm{H}^{\prime}$ to fit in the DNA major groove. On the other hand, human $\mathrm{TFIIB}_{\mathrm{C}}$ uses residues in $\mathrm{H}^{\prime}$, the recognition helix of the HTH motif, to make base-specific contacts with the major groove $(29,33)$. tTFIIB $_{C}$ contains similar residues in $\mathrm{H}^{\prime}$ (Thr-292, Lys-293, Asn-295, and Lys-296) that may function analogously in DNA recognition.

A major portion of the neutral electrostatic surface of $\mathrm{tTFIIB}_{\mathrm{C}}$ derives from surface residues on helix $\mathrm{H} 5$. In the human TFIIB $_{\mathrm{C}}$ TBP/DNA complex, H5 stabilizes the TBP-induced deformation of

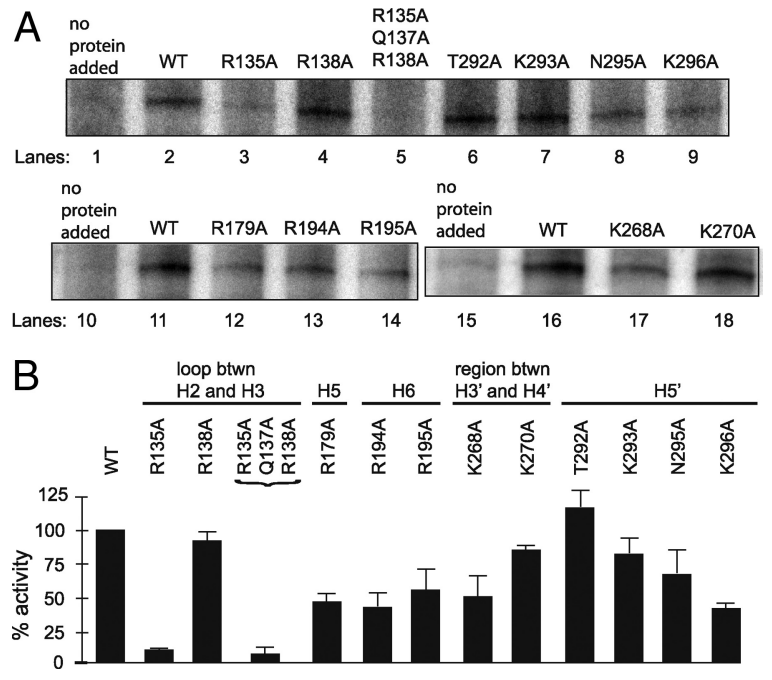

Fig. 4. Activity of tTFIIB mutants with alanine substitutions in in vitro SL RNA transcription assays. (A) Transcription activity in tTFIIB-depleted extracts (lanes 1 and 10) was restored upon addback of wild-type (WT) tTFIIB (lanes 2 and 11). In the loop linking $\mathrm{H} 2$ and $\mathrm{H} 3$, the R138A mutant had near WT activity (lane 4), but both R135A and the triple mutant nearly abolished activity. Mutations in $\mathrm{H} 5$ and $\mathrm{H} 6$ (R179A, R194A, R195A; lanes $12-14$ ) reduced activity $\approx 50 \%$. In the linker between $\mathrm{H}^{\prime}$ and $\mathrm{H}^{\prime}, \mathrm{K} 168 \mathrm{~A}$ also reduced activity $\approx 50 \%$ (lane 17 ), while K270 had near WT activity (lane 18). In helix H5', T292A had increased activity (lane 6), while K293A, N295A, and K296A toward the center of the helix had reduced activity (lane 7-9). (B) Histogram represents averages of 3 replicates for each mutant. Error bars indicate 1 standard deviation.

the DNA through interactions between Lys-189 and Arg-193 and the DNA phosphate backbone (29). In TFFIIB $_{C}$, the corresponding region of $\mathrm{H} 5$ does not contain basic residues. However, the basic residues adjacent to this region, including Arg-179 in H5 and Arg-194 and Arg-195 in H6, may assist in stabilizing the trypanosome $\mathrm{TFIIB}_{\mathrm{C}}$ TBP/DNA complex.

Activity of tTFIIB Mutants by in Vitro Transcription. To determine if specific residues in $\mathrm{tTFIIB}_{\mathrm{C}}$ are important for function, we assayed variants of full-length tTFIIB for their ability to support transcription. We made alanine substitutions in Arg-135 and Arg-138 and the triple substitution Arg-135/Gln-137/Arg-138 in the loop linking $\mathrm{H} 2$ and $\mathrm{H} 3$. We also made single alanine substitutions in Arg-179 in H5, Arg-194 and Arg-195 in H6, Lys-268 and Lys-270 in the linker between $\mathrm{H}^{\prime}{ }^{\prime}$ and $\mathrm{H}^{\prime}{ }^{\prime}$, and Thr-292, Lys-293, Asn-295, and Lys-296 in H5' (Fig. S4).

Single alanine substitutions in the loop linking $\mathrm{H} 2$ and $\mathrm{H} 3$ reduced activity minimally in the case of Arg- 138 but decreased activity to $\approx 10 \%$ in the case of Arg-135 (Fig. 4). The triple variant, containing alanine substitutions of Arg-138, Arg-135 along with Gln-137, reduced activity to $\approx 7 \%$. The single mutation in helix $\mathrm{H} 5$ reduced activity to $\approx 50 \%$. Mutations of basic residues near the center of helix $\mathrm{H}^{\prime}$ ' had a more severe effect on transcription than those near the N-terminal edge of the helix. Specifically, Lys-296 reduced activity to $\approx 40 \%$, whereas Lys- 293 reduced activity to $\approx 80 \%$. The Asn-295 to Ala mutation reduced activity to $\approx 65 \%$ and the Thr-292 to Ala mutation appeared to stimulate tTFIIB activity. Overall, mutations in trypanosomespecific regions reduced activity of tTFIIB. The mutations of basic residues in $\mathrm{H} 6$ and Lys-268 in the linker between $\mathrm{H}^{\prime}$ and $\mathrm{H} 4$ ' reduced activity to $40-50 \%$. Mutation of Lys-270 had only a small effect $(\approx 85 \%$ activity). Taken together, these data suggest that basic residues within the loop linking $\mathrm{H} 2$ and $\mathrm{H} 3$, the helices $\mathrm{H} 5$ and $\mathrm{H}^{\prime}$, and in the trypanosome-specific regions ( $\mathrm{H} 6$ and the linker between $\mathrm{H}^{\prime}{ }^{\prime}$ and $\mathrm{H}^{\prime}$ ) are important for in vitro 
transcription, probably by stabilizing tTFIIB interactions at the SL RNA gene promoter.

\section{Discussion}

This work describes the high-resolution structure of a trypanosome transcription factor. The structure of trypanosome TFIIB $_{C}$ shares important features with its human counterpart. tTFIIB $_{C}$ contains 2 cyclin folds that align well to human TFIIB $_{C}$ even though the second module was not apparent in TTFIIB $_{C}$ from primary sequence information. In $T$. brucei, the loop between helices $\mathrm{H} 2$ and $\mathrm{H} 3$ is functionally important, as is the corresponding recognition loop in the human protein. Specifically, mutation of basic amino acids in this region completely compromised TFIIB function in the human and trypanosome proteins. Thus, our work supports a fundamental role of the loop between helices $\mathrm{H} 2$ and $\mathrm{H} 3$ in transcription.

Trypanosome TFIIB $_{C}$ deviates in potentially significant ways from its human counterpart. The 2 cyclin folds are connected by a helix (H6) rather than a random coil, and in between helices $\mathrm{H}^{\prime}$ ' and $\mathrm{H}^{\prime}$ ', there is an additional helix ( $\left.\mathrm{H}^{\prime}{ }^{\prime} \mathrm{A}\right)$ followed by a disordered 13-residue linker. Importantly, mutations in these regions reduced transcriptional activity of tTFIIB, indicating that these regions play a role in tTFIIB function. Moreover, the presence of a potentially protease-sensitive linker between $\mathrm{H}^{\prime}$ and $\mathrm{H}^{\prime}$ ' may explain why tTFIIB lacks a protease-resistant core. In addition to the 13 disordered residues in the linker, the C-terminal 32 residues of the TTFIIB $_{C}$ structure are also disordered. These regions are common to all trypanosome TFIIB proteins sequenced thus far, and while the sequences vary in length between each protein, they have similar amino acid composition. It is likely that these unstructured segments contain short, peptide motifs involved in recognizing DNA or other trypanosome-specific protein partners, possible adopting secondary structure upon binding. These segments will be the object of future studies.

Trypanosome TFIIB lacks the conserved Gln and Arg residues in helices $\mathrm{H}^{\prime}$ ' and $\mathrm{H}^{\prime}$ ' that stabilize the interaction of HTH motifs with the DNA major groove. In addition, tTFIIB $\mathrm{H}^{\prime}{ }^{\prime}$ has an additional helical turn that would hinder major groove binding. Mutations in H5' (Thr-292 and Asn-295) did not knock out function as they did in the corresponding residues (Val-283 and Arg-286) in human TFIIB (33). These traits in the trypanosome protein may be related to the lack of a $\mathrm{BRE}^{\mathrm{U}}$ in the SL RNA gene promoter. In human TATA box-containing promoters, TFIIB binding to the BRE ${ }^{\mathrm{U}}$ directs assembly of the preinitiation complex in the correct orientation. The majority of gene promoters do not contain a TATA box or an associated $\mathrm{BRE}^{\mathrm{U}}$, thus they rely on multiple interactions from transcription-associated factors for preinitiation complex orientation. In trypanosome TFIIB, the linker between $\mathrm{H}^{\prime}$ ' and $\mathrm{H}_{4}$ ', as well as the C-terminal extension, may recruit factors that help determine preinitiation orientation at the SL RNA gene promoter.

tTFIIB $_{C}$ contains a large neutral surface in place of the highly basic surface on the human protein (Fig. 3). The large neutral surface might be required to stabilize TBP binding to DNA. Whereas human TBP has 4 phenylalanines that deform the TATA box upon binding, only 2 phenylalanines are present in trypanosome TBP $(10,34)$. Thus, tTFIIB may help stabilize weak tTBP-promoter interactions. In support of this argument, our previous studies showed that tTBP and tTFIIB tightly associate (14).

In well-studied eukaryotic transcription, the basic surface of TFIIB specifically binds at gene promoters to orient RNAP-II so that transcription proceeds in the correct direction. The lack of an extensive basic surface on trypanosome TFIIB may reflect a relaxed specificity of tRNAP-II orientation at promoters in trypanosomes. Most trypanosome protein-encoding genes are transcribed in large arrays that appear to initiate bidirectionally from strand switch regions (35-37). Weak specificity of preinitiation complex orientation could cause bidirectional transcription from strand switch regions. Thus, trypanosome TFIIB would be relieved of the function to orient tRNAP-II for unidirectional transcription.

\section{Methods}

Protein Expression and Purification. Full-length T. brucei TFIIB (tTFIIB), the C-terminal domain (tTFIIB $\mathrm{B}_{\mathrm{C}}$, and variants were recombinantly expressed and purified from $E$. coli as $\mathrm{His}_{6}-\mathrm{MBP}$ fusion proteins following the protocol of Tropea et al. (38). TFIIB and variants were purified by sequential affinity and gel filtration chromatography. For $\operatorname{TTFIIB}_{C}$, the latter step was replaced by ion exchange chromatography. The selenomethionine labeled TTFIIB $_{C}$ (SeMettTFIIB $B_{C}$ ) was expressed as described (39) and purified as described above for

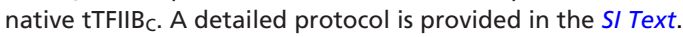

Limited Proteolysis. tTFIIB $(20 \mu \mathrm{M})$ was digested with trypsin or subtilisin in 50 $\mathrm{mM}$ Tris- $\mathrm{HCl}, \mathrm{pH} 8.0,50 \mathrm{mM} \mathrm{NaCl}$ at $25^{\circ} \mathrm{C}$ for $1 \mathrm{~h}$ in $50 \mu \mathrm{L}$ with an enzyme: substrate molar ratio of 1:400. Aliquots $(10 \mu \mathrm{L})$ were removed at 5-, 10-, 15-, 30-, 60-min intervals, and the reaction in the aliquot was stopped by addition of $4 \%$ SDS, $100 \mathrm{mM}$ DTT, $20 \mathrm{mM}$ EDTA, $2 \mathrm{mM}$ PMSF, and flash-freezing. Samples were heated to $95^{\circ} \mathrm{C}$ for $10 \mathrm{~min}$ and analyzed by SDS/PAGE.

Gel Filtration and Chemical Denaturation. Gel filtration was performed on a Sephacryl S-200 column equilibrated in $25 \mathrm{mM}$ Tris- $\mathrm{HCl}, \mathrm{pH} 8.0,150 \mathrm{mM}$

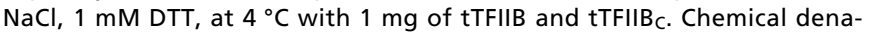
turation experiments monitoring tryptophan fluorescence were performed at $25^{\circ} \mathrm{C}$ (QuantaMaster spectrofluorometer; Photon Technology International). Samples of $3 \mu \mathrm{M}$ tTFIIB or tTFIIB $_{\mathrm{C}}$ were prepared at each guanidinium chloride $(\mathrm{GdmCl})$ concentration in $25 \mathrm{mM}$ potassium phosphate, pH 7.5, $200 \mathrm{mM} \mathrm{KCl}$, and $1 \mathrm{mM} \mathrm{DTT}$ and incubated overnight at room temperature. Fluorescence emission spectra were measured by exciting the samples at $265 \mathrm{~nm}$ and recording spectra from 300 to $400 \mathrm{~nm}$ (0.1-s integration time) and the center-of-mass of the spectral peak was calculated. The $\mathrm{GdmCl}$ concentration was determined by refractive index using a Bausch \& Lomb refractometer. $\mathrm{GdmCl}$ denaturation of tTFIIB or tTFIIB $_{C}$ was reversible as judged by the recovery of center-of-mass from dilution of the $4 \mathrm{M} \mathrm{GdmCl}$ sample to $2 \mathrm{M} \mathrm{GdmCl}$. Curve fitting was performed in Kaleidagraph (Synergy Software).

Immunodepletion and in Vitro Transcription. $T$. brucei nuclear extracts were prepared as described $(11,40)$. Immunodepletion and in vitro transcription assays were performed as described (14) with the following exceptions. Transcription was monitored by detecting radiolabeled run-off transcript (172 bases) from a linearized plasmid rather than by primer extension. The plasmid pJP10, which contains the -125 - to +120 region of the SL RNA gene was linearized with ECoRV. In the reaction, $250 \mu \mathrm{M} \mathrm{CTP}$ and GTP, $1 \mathrm{mM} \mathrm{ATP}$, and $10 \mu \mathrm{Ci}$ alpha- ${ }^{32} \mathrm{P}$-labeled UTP were incubated at $28^{\circ} \mathrm{C}$ with other reaction components for $10 \mathrm{~min}$, after which cold UTP was added to a final concentration of $50 \mu \mathrm{M}$. The potassium glutamate concentration in the reaction was $130 \mathrm{mM}$.

Structure Determination of tTFIIBc. Crystals of native TTFIIB $_{C}$ were obtained by vapor diffusion against a reservoir containing $0.1 \mathrm{M}$ Bis-Tris, $\mathrm{pH} 5.5$, and $24 \%$ polyethylene glycol 3350 . SeMet- TFIIB $_{C}$ protein crystallized in the same space group $\left(\mathrm{P}_{3} 2_{2}{ }_{2}\right)$ but required slightly higher concentrations of polyethylene glycol $(32 \%)$ to form. The structure was solved using 3-wavelength multiple anomalous dispersion from data collected at National Synchrotron Light Source beamline $X 29$ and refined against a native data set collected at the PHRI X-Ray Crystallography Core Facility. The final model consists of residues 94-261 and 275-313, 56 water molecules, and an ethylene glycol molecule, with values of $R_{\text {work }}=0.204$ and $R_{\text {free }}=0.249$. Details are provided in SI Text, and data collection and refinement statistics are given in Table S1 and Table S2.

ACKNOWLEDGMENTS. We thank the staff of beamline X29 at the National Synchrotron Light Source; Mary Ann Gawinowicz at the Columbia University Protein Core Facility; Tara Kurumaddali for assistance with site-directed mutagenesis; and Karl Drlica, David Dubnau, Phil Jeffrey, Leonard Mindich, Matthew Neiditch, David Perlin, and Issar Smith for helpful discussions. This work was supported by National Institutes of Health Grant Al-29478 (to V.B.), American Heart Association Fellowship 0425791T (to J.B.P.), American Heart Association Scientist Development Grant 0735532T (to A.D.), and Ministerio de Educacion y Ciencia (Spain) Grant CTQ2007-68014-C02-02 (to D.V.L.). 
1. Simpson AG, Stevens JR, Lukes J (2006) The evolution and diversity of kinetoplastid flagellates. Trends Parasitol 22:168-174.

2. Despommier DD, Gwadz RW, Hotez PJ, Knirsch CA (2005) Parasitic Diseases (Apple Trees Productions, New York, NY).

3. Clayton CE (2002) Life without transcriptional control? From fly to man and back again EMBO J 21:1881-1888.

4. Palenchar JB, Bellofatto V (2006) Gene transcription in trypanosomes. Mol Biochem Parasitol 146:135-141.

5. Liang XH, Haritan A, Uliel S, Michaeli S (2003) trans and cis splicing in trypanosomatids: Mechanism, factors, and regulation. Eukaryot Cell 2:830-840.

6. Gilinger G, Bellofatto V (2001) Trypanosome spliced leader RNA genes contain the first identified RNA polymerase II gene promoter in these organisms. Nucleic Acids Res 29:1556-1564.

7. Campbell DA, Thomas S, Sturm NR (2003) Transcription in kinetoplastid protozoa: Why be normal? Microbes Infect 5:1231-1240.

8. Das A, Bellofatto V (2003) RNA polymerase II-dependent transcription in trypanosomes is associated with a SNAP complex-like transcription factor. Proc Natl Acad Sci USA 100:80-85.

9. Schimanski B, Laufer G, Gontcharova L, Gunzl A (2004) The Trypanosoma brucei spliced leader RNA and rRNA gene promoters have interchangeable TbSNAP50-binding elements. Nucleic Acids Res 32:700-709.

10. Ruan JP, Arhin GK, Ullu E, Tschudi C (2004) Functional characterization of a Trypanosoma brucei TATA-binding protein-related factor points to a universal regulator of transcription in trypanosomes. Mol Cell Biol 24:9610-9618.

11. Das A, et al. (2005) Trypanosomal TBP functions with the multisubunit transcription factor tSNAP to direct spliced-leader RNA gene expression. Mo/ Cell Bio/ 25:7314-7322

12. Schimanski B, Nguyen TN, Gunzl A (2005) Characterization of a multisubunit transcrip tion factor complex essential for spliced-leader RNA gene transcription in Trypanosoma brucei. Mol Cell Biol 25:7303-7313.

13. Schimanski B, Brandenburg J, Nguyen TN, Caimano MJ, Gunzl A (2006) A TFIIB-like protein is indispensable for spliced leader RNA gene transcription in Trypanosoma brucei. Nucleic Acids Res 34:1676-1684.

14. Palenchar JB, Liu W, Palenchar PM, Bellofatto V (2006) A divergent transcription factor TFIIB in trypanosomes is required for RNA polymerase II-dependent spliced leader RNA transcription and cell viability. Eukaryot Cell 5:293-300.

15. Thomas S, Yu MC, Sturm NR, Campbell DA (2006) A non-universal transcription factor? The Leishmania tarentolae TATA box-binding protein LtTBP associates with a subset of promoters. Int J Parasitol 36:1217-1226.

16. Lecordier L, et al. (2007) Characterization of a TFIIH homologue from Trypanosoma brucei. Mol Microbiol 64:1164-1181.

17. Lee JH, Nguyen TN, Schimanski B, Gunzl A (2007) Spliced leader RNA gene transcription in Trypanosoma brucei requires transcription factor TFIIH. Eukaryot Cell 6:641-649.

18. Lee JH, Jung HS, Gunzl A (2009) Transcriptionally active TFIIH of the early-diverged eukaryote Trypanosoma brucei harbors two novel core subunits but not a cyclinactivating kinase complex. Nucleic Acids Res 37:3811-3820.

19. Deng W, Roberts SG (2007) TFIIB and the regulation of transcription by RNA polymerase II. Chromosoma 116:417-429.

20. Barberis A, Muller CW, Harrison SC, Ptashne M (1993) Delineation of two functional regions of transcription factor TFIIB. Proc Natl Acad Sci USA 90:5628-5632.
21. Malik S, Lee DK, Roeder RG (1993) Potential RNA polymerase II-induced interactions of transcription factor TFIIB. Mol Cell Biol 13:6253-6259.

22. Chen HT, Legault P, Glushka J, Omichinski JG, Scott RA (2000) Structure of a (Cys3His) zinc ribbon, a ubiquitous motif in archaeal and eucaryal transcription. Protein Sci 9:1743-1752.

23. Zhu W, et al. (1996) The N-terminal domain of TFIIB from Pyrococcus furiosus forms a zinc ribbon. Nat Struct Biol 3:122-124.

24. Bushnell DA, Westover KD, Davis RE, Kornberg RD (2004) Structural basis of transcription: An RNA polymerase II-TFIIB cocrystal at 4.5 Angstroms. Science 303:983-988.

25. Bagby S, et al. (1995) Solution structure of the C-terminal core domain of human TFIIB: Similarity to cyclin A and interaction with TATA-binding protein. Cell 82:857-867.

26. Nikolov DB, et al. (1995) Crystal structure of a TFIIB-TBP-TATA-element ternary complex. Nature 377:119-128.

27. Kosa PF, Ghosh G, DeDecker BS, Sigler PB (1997) The 2.1-A crystal structure of an archaeal preinitiation complex: TATA-box-binding protein/transcription factor (II)B core/TATA-box. Proc Natl Acad Sci USA 94:6042-6047.

28. Littlefield O, Korkhin Y, Sigler PB (1999) The structural basis for the oriented assembly of a TBP/TFB/promoter complex. Proc Natl Acad Sci USA 96:13668-13673.

29. Tsai FT, Sigler PB (2000) Structural basis of preinitiation complex assembly on human pol II promoters. $E M B O J$ 19:25-36.

30. Romero P, et al. (2001) Sequence complexity of disordered protein. Proteins 42:38-48.

31. Holm L, Kaariainen S, Rosenstrom P, Schenkel A (2008) Searching protein structure databases with DaliLite v. 3. Bioinformatics 24:2780-2781.

32. Mohan A, et al. (2006) Analysis of molecular recognition features (MoRFs). J Mol Biol 362:1043-1059.

33. Lagrange T, Kapanidis AN, Tang H, Reinberg D, Ebright RH (1998) New core promoter element in RNA polymerase II-dependent transcription: Sequence-specific DNA binding by transcription factor IIB. Genes Dev 12:34-44.

34. Juo ZS, et al. (1996) How proteins recognize the TATA box. J Mol Biol 261:239-254

35. Martinez-Calvillo S, et al. (2003) Transcription of Leishmania major Friedlin chromo some 1 initiates in both directions within a single region. Mol Cell 11:1291-1299.

36. Respuela P, Ferella M, Rada-Iglesias A, Aslund L (2008) Histone acetylation and methylation at sites initiating divergent polycistronic transcription in Trypanosoma cruzi. I Biol Chem 283:15884-15892.

37. Siegel TN, et al. (2009) Four histone variants mark the boundaries of polycistronic transcription units in Trypanosoma brucei. Genes Dev 23:1063-1076.

38. Tropea JE, Cherry S, Nallamsetty S, Bignon C, Waugh DS (2007) A generic method for the production of recombinant proteins in Escherichia coli using a dual hexahistidinemaltose-binding protein affinity tag. Methods Mol Biol 363:1-19.

39. Van Duyne GD, Standaert RF, Karplus PA, Schreiber SL, Clardy J (1993) Atomic structures of the human immunophilin FKBP-12 complexes with FK506 and rapamycin. J Mol BiO 229:105-124.

40. Huie JL, He P, Bellofatto V (1997) In vitro transcription of the Leptomonas seymouri SL RNA and U2 snRNA genes using homologous cell extracts. Mol Biochem Parasitol 90:183-192. 